\title{
Germanica
}

\section{Au nom du père}

Le voyage, réalité et imaginaire dans la trilogie d'Ib Michael

Im Namen des Vaters. Wirkliches und imaginäres Reisen in der Romantrilogie

von Ib Michael

\section{Kirsten-Lai Jensen}

\section{(2) OpenEdition}

\section{Journals}

Édition électronique

URL : http://journals.openedition.org/germanica/2200

DOI : 10.4000/germanica.2200

ISSN : 2107-0784

Éditeur

Université de Lille

\section{Édition imprimée}

Date de publication : 31 décembre 2001

Pagination : 135-147

ISBN : 9782913857063

ISSN : 0984-2632

\section{Référence électronique}

Kirsten-Lai Jensen, «Au nom du père », Germanica [En ligne], 29 | 2001, mis en ligne le 20 septembre 2013, consulté le 06 octobre 2020. URL : http://journals.openedition.org/germanica/2200 ; DOI :

https://doi.org/10.4000/germanica.2200

Ce document a été généré automatiquement le 6 octobre 2020.

(c) Tous droits réservés 


\title{
Au nom du père
}

\author{
Le voyage, réalité et imaginaire dans la trilogie d'Ib Michael \\ Im Namen des Vaters. Wirkliches und imaginäres Reisen in der Romantrilogie \\ von Ib Michael
}

Kirsten-Lai Jensen

Un soir de novembre, vers la fin du siècle, un homme arrive en gare de Roskilde (La fille vanille, 1991, 11). Cet homme-là, c'est le narrateur des trois romans : La fille vanille (1991, traduit en français en 1996), Le douzième cavalier (1993) et Lettre à la lune (1995), qui forment la trilogie signée par Ib Michael, écrivain danois (1943-). Auteur d'une œuvre riche, Ib Michael s'est depuis longtemps fait connaître au Danemark avec des romans tels que Le troubadour et la fille du vent (1984, traduit en français en 1989) et Kilroy, Kilroy (1989, traduit en français en 1993), des textes qui nous mènent loin du Danemark. Dans la première moitié des années 1990, il connaît un succès populaire sans précédent avec sa trilogie avec laquelle il revient dans son pays natal. Ici, l'exotisme renoue avec le Danemark si familier de ses lecteurs. L'univers des trois romans demeure cependant profondément marqué par les nombreux voyages de l'auteur.

Dans la presse, on a voulu souligner le caractère autobiographique de cette trilogie. Il n'est cependant pas question de confondre l'auteur et le narrateur. Dans une interview donnée à l'occasion de la sortie du Douzième cavalier, Ib Michael déclare : Dans ce livre, je crée une sorte de métafiction où je suis moi-même identique au personnage du narrateur [...]. Pour que tout soit bien clair : je n'écris pas sur ma propre famille. Ce que j'ai fait, c'est une fiction sur La Famille et son importance (Ce n'est pas la réalité qui est étrange, Information, 2.4.1993). À cela s'ajoute l'exotisme, les côtés fantastiques et magiques du récit, des côtés qui sont révélateurs pour l'univers des romans et pour toute l'ambiance qui s'en dégage. Fantaisies, rêves, fantômes et visions se mêlent à la réalité - et sont pris en compte comme s'ils étaient bien réels.

3 En toute première page du premier roman, La fille vanille, le narrateur revient à Roskilde, sa ville natale, à l'occasion de l'enterrement de son père. Dès le prologue, nous comprenons que cet homme revient d'un long voyage, d'une longue quête. Mais en fait, le voyage ne fait que commencer. De la rencontre avec sa ville natale naît le 
souvenir de son enfance alors qu'il était « le prince héritier » de la dynastie familiale ; ce souvenir s'impose à lui et le tiendra enfermé dans sa chambre d'hôtel jusqu'à ce qu'il se décide à affronter tous les fantômes familiaux. Sa chambre d'hôtel en est hantée et à chaque fois que le narrateur essaie de quitter sa chambre et par là, de quitter ses souvenirs, quelque chose se produit: un tableau se décroche du mur, des photos familiales tombent par terre juste devant lui, etc., réveillant à chaque fois d'autres souvenirs, qui l'entraînent dans le passé. Sa confrontation avec ses souvenirs nous emmènera non seulement à travers l'enfance (La fille vanille) et la jeunesse (Lettre à la lune) du narrateur, mais également des siècles en arrière pour découvrir les racines douloureuses et violentes de sa famille (Le douzième cavalier). Cette trilogie représente donc un long cheminement spirituel qui sera marqué par de nombreux autres voyages, tant fantastiques que réels.

\section{Voyages imaginaires et découvertes}

4 Fils d'un commerçant parvenu, notre héros a vécu une enfance matériellement aisée. Ses premiers voyages se font aux alentours de la maison familiale, le jardin semble immense, la maison est un labyrinthe, tout ressemble à une grande aventure excitante et un peu inquiétante: il arrive parfois que je m'aventure jusqu'au fond du jardin, où un grand portillon ferme l'accès à la rue. Là commence le pays interdit (La fille vanille, 21). Lentement, son monde s'agrandit et avant même de sortir du jardin, il réveille son premier fantôme, un squelette découvert sous le châtaignier du jardin, un Espagnol. Un cavalier noir, (26) qui deviendra pour lui un compagnon de guerre contre sa nourrice, avant de se confondre avec le soldat espagnol, son aïeul évoqué si souvent par sa grandmère. Sa grande découverte suivante est la malle remplie de lettres écrites par l'oncle Viggo, le frère de sa grand-mère. Habité par le gène voyageur de la famille, ce dernier a d'abord passé de nombreuses années en mer comme capitaine d'un bateau. Ensuite il a régné en monarque absolu sur une île du Pacifique. D'instinct, l'enfant s'identifie à lui : Dans une atmosphère de conspiration, je [...] prête le serment d'être, moi aussi, une brebis galeuse, (41)

5 Par ailleurs, c'est aussi du côté de sa grand-mère et de sa sœur Lulu que le narrateur cherche et élabore les éléments qui vont peupler son monde imaginaire et ses voyages dans le fantastique. Il écoute avec attention et intérêt les histoires que lui raconte sa grand-mère à propos de l'arrivée au Danemark du soldat espagnol avec les troupes de Napoléon et des aventures de son frère Viggo dont les lettres sont intégrées au roman. Quant à sa petite sœur, il l'adore. Il joue bien sûr le rôle du grand frère protecteur et c'est avec elle qu'il va partager et élargir son univers fantastique. Il la voit comme l'ange que seul un miracle a fait revenir sur terre après que la poliomyélite a failli l'emporter. Deux autres miracles se produisent dans la vie du petit garçon : suite aux nombreuses aventures amoureuses de son mari, sa mère se révolte et part en Afrique avec des amis, notamment avec le colonel Leschley que l'enfant admire et dont il rêve secrètement d'être le fils. Notre héros croit sa mère partie pour toujours, absorbée par le continent africain, et quand elle revient enfin, c'est un miracle pour lui, c'est la réponse à ses prières. Ce retour déclenche le dernier des trois miracles: dans le bonheur de revoir enfin sa mère, la petite Lulu, grâce à sa volonté de fer, marche pour la première fois - contre toute espérance et contre l'avis des médecins. La réalité du roman permet donc les miracles, le fantastique fait pratiquement partie de la vie 
quotidienne de l'enfant et il y existe même une certaine complicité entre l'enfant, les fantômes et les anges qui peuplent son monde imaginaire. L'enfant invente des histoires pour sa sœur, pour la protéger et pour se protéger soi-même contre le mal, contre la mort :

Il n'y a plus à avoir peur, maintenant que je dois veiller sur elle [...]. On m'a confié la tâche d'appeler [...] si Lulu arrêtait de nouveau de respirer [...]. Je suis terrorisé et je guette très longtemps. À la fin je n'entends plus que le bruit de mon cœur solitaire qui en cognant devient de plus en plus bruyant dans le silence. J'appelle à travers la pièce... « Lulu ! » [...]. Tant qu'elle me répond, je n'ai pas besoin de me demander si elle respire. Tout doucement, allongé dans le noir, je grandis, tandis que je lui parle. [...], (119).

6 C'est à travers ses histoires, à travers son monde imaginaire qu'il va arriver à connaître, à façonner et à comprendre à sa manière le monde des adultes. Sa conception du monde ne correspond pas à celle des adultes, l'enfant prend tout très au sérieux. Un soir, par exemple, il entend son père se plaindre du bifteck : c'est de la vraie semelle de chaussure, (28). Alors l'enfant prend le bifteck pour s'en servir plus tard: Comme on me dit toujours que j'use mes talons [...], j'ai essayé de le fixer sur ma chaussure [...], (31).

7 La compagne fidèle des voyages imaginaires des deux enfants est la « fille vanille ». Elle figure sur les sachets de sucre vanillé et réunit aux yeux des deux enfants l'exotisme et la sécurité du monde connu. Elle est la femme polynésienne de l'oncle Viggo, la princesse de l'île, elle réunit la beauté, les aventures et la confiance en soi :

Quand la maison devient trop grande, je me réfugie dans le garde-manger. [...]. Dans ce réduit habite la fille vanille avec laquelle je chuchote dans la langue des nègres. Elle sait où se trouve l'oncle Viggo et où est enterré le trésor. Son visage [...] orne les sachets de vanille en poudre [...]. Il faut déchirer un coin et verser la poudre dans la main. Quand on y pose la langue, ça devient une colle sombre qui réveille dans la bouche un rêve de pays lointains. [...] La fille des sachets de vanille est la reine des épices. [...]. Elle est la nourrice de mon monde secret, car avec elle il ne faut jamais se laver et ses mains transforment la douleur en velours, (60-61).

Les voyages imaginaires que les deux enfants effectuent avec elle deviennent rapidement un moyen d'échapper aux autres pour une découverte de soi, un moyen de supporter et de transformer la réalité, de combler le vide, de fuir la peur.

\section{Jeunesse : départs et découvertes}

9 La fille vanille est dédiée à l'enfance du narrateur. Il est significatif qu'il n'aborde son adolescence, sa jeunesse qu'après le long détour du Douzième cavalier. Il évite le plus longtemps possible de se confronter aux douloureux souvenirs d'un passé longtemps réprimé. Mais à la fin du Douzième cavalier, il est obligé d'y faire face, il remémore l'été passé avec Maiken, son premier grand amour que son père ne manque pas de lui voler. Ce fut un désastre pour le jeune garçon mais l'homme mûr qu'est notre narrateur parvient maintenant, avec le recul des années, à affronter cette douleur. La suite et surtout la fin de la trilogie nous en dévoileront le résultat.

Lettre à la lune reprend le fil de La fille vanille. En pleine adolescence, le narrateur affronte de plus en plus souvent son père. Ce dernier représente une image masculine à laquelle notre héros n'arrive pas à s'identifier. Entre eux, il est souvent question de rivalité, notamment dans deux domaines : l'équitation et les relations amoureuses. Fier de son fils, le père se vante sans cesse mais il ne manque pas non plus de remettre à sa 
place ce fils qui menace sa position. Le garçon est au début du roman partagé entre admiration et dégoût, tout comme dans La fille vanille mais, au fil des pages, il prend ses distances par rapport à son père et s'aventure dans une direction opposée. La rencontre avec Olaf, un ami d'enfance, va déclencher la rupture avec le monde paternel et donner libre cours à une nouvelle orientation. Olaf servira de médiateur au narrateur pour le faire pénétrer dans le monde alternatif et artistique. Auprès de son ami, le narrateur trouve enfin le complice qui lui a tant manqué dans le monde masculin. Dans sa révolte contre les valeurs familiales, contre son père surtout, le jeune homme en arrive jusqu'à tromper son père en couchant avec sa maîtresse, c'est là sûrement la vengeance pour Maiken. Mais c'est avec dégoût que le jeune protagoniste recourt aux moyens utilisés par son père : J'ai envie mais pas d'elle. Ça donne un mauvais goût dans la bouche et j'arrive à peine à l'exécution, (Lettre à la lune, 140). C'est finalement l'absence qui sera l'arme la plus efficace contre le père. Le narrateur quitte la maison familiale, il s'installe à Copenhague avec Olaf. Occupé par ses propres projets, il ne se soucie guère de ceux de son père. Celui-ci agrandit son entreprise et y fait installer un bureau pour "Junior ». Mais lorsque l'inauguration arrive, le fils ne vient pas (il est en route pour Venise) et les rêves du père se brisent. Avec Olaf, «Junior " découvre un monde nouveau, ses rêves d'artiste se révèlent. Il écrit des poèmes - du moins, il essaie d'en écrire ; quant à Olaf, il peint. Bien sûr, il leur faut des petits boulots pour survivre mais cela n'a pas beaucoup d'importance pour eux. Leur monde tourne autour d'autres buts: ils veulent aller à Venise, la cité magique, en compagnie de Kate, une amie d'Olaf. Souffrant d'une grave schizophrénie, elle est victime d'une mauvaise expérience avec du L.S.D. - nous sommes dans les années soixante-dix. Les deux garçons la perçoivent comme un être féerique et fragile dont il faut prendre soin pour sauvegarder sa vie, sa magie et son bonheur. Voilà la priorité principale pour les deux jeunes gens. C'est pourquoi notre narrateur va découvrir un aspect de l'univers masculin qu'il n'a pas encore connu : la solidarité avec Olaf remplace la rivalité qu'il a toujours vécue avec son père.

Dans Lettre à la lune, il est question de découvertes. Immature au début du roman, le narrateur se développe tout au long de celui-ci, il se découvre graduellement. Ce développement permet au narrateur une conscience croissante dans ses réactions, ses gestes et actes, et également au niveau de sa façon de concevoir le monde. Le côté imaginaire est toujours important pour lui et il arrive souvent qu'un détail déclenche tout un film dans sa tête. Mais il ne découvre pas seulement son propre monde. Il y a également des voyages dans le monde de la drogue, et les voyages imaginaires avec Kate qui ne cesse de mettre en scène la vie des deux garçons, le décor de leur appartement, leur style vestimentaire, etc. Kate est en perpétuel changement. Tu ne sais jamais qui elle est. Son armoire ressemble à une garde-robe de théâtre, (189). Ensuite et surtout, il y a de nombreux voyages à l'étranger: d'abord à Malmö en Suède avec l'équipe d'équitation, ensuite un voyage en solitaire à Paris, renouvelé après son bac l'année suivante, et finalement le grand départ pour Venise avec Olaf. Bien évidemment, il est question de découvrir d'autres cultures, d'autres mœurs, mais, pour le jeune homme, il est surtout question de se découvrir soi-même, à travers les autres, à travers les relations qu'il entretient avec eux. Ses voyages à l'étranger soulèvent toujours un même intérêt : la découverte de l'autre sexe.

12 Les relations amoureuses s'avèrent tout au long du roman difficiles pour le jeune homme. Certes, il a beaucoup d'aventures féminines, mais, quand c'est vraiment important pour lui, il échoue. Son dernier échec a lieu à Venise. N'arrivant plus à dissimuler les sentiments qu'il éprouve pour Kate, il en fait part à Olaf qui lui cède la 
place non sans réticence. Mais il n'arrive pas à faire face aux personnalités multiples de Kate, son côté morbide, son envie de mourir. Puis Kate disparait pour être retrouvée morte suicidée quelques jours plus tard. C'est la fin tragique de l'aventure vénitienne, fin également du récit de la jeunesse du narrateur qui laisse la place à l'homme qui se trouve bien des années plus tard enfermé dans une chambre d'hôtel à Roskilde.

\section{Voyage dans l'histoire}

13 La confrontation avec son passé le plus douloureux n'a pas été facile pour le narrateur, bien au contraire, elle nécessite un grand détour qui à lui seul forme un tome entier de la trilogie. Dans Le douzième cavalier, le narrateur remonte dans l'histoire familiale et également dans l'Histoire avec un grand H. Les deux tiers du roman se déroulent sans même évoquer le narrateur, il n'est plus le personnage principal du récit. Le douzième cavalier dépeint les inégalités sociales criantes du Danemark au début du XIX siècle. À cela s'ajoute la description du Mexique, un monde étrange qui nous est montré à travers les yeux des Danois pour lesquels le pays est fortement empreint d'exotisme. Les scènes et les couleurs du Douzième cavalier sont violentes et nous emmènent loin de la sécurité du monde enfantin vécu dans La fille vanille. Nous quittons l'enfance du narrateur pour nous retrouver en compagnie de Gomez, un soldat espagnol venu au Danemark en 1808 avec les troupes de Napoléon. Confronté au froid de l'hiver scandinave, il finit par faire brûler le château de Kolding. Il séduit ensuite une jeune servante, viole une jeune femme noble et de ces deux rencontres naissent deux enfants qui fondent sa descendance danoise. Après le viol, Gomez est condamné et emprisonné. Dans l'indescriptible crasse des geôles de l'époque, il disparaît dans l'oubli, dans le néant et sa renaissance - difficile - s'opère grâce au pasteur de la prison qui s'intéresse à l'étranger :

Gomez apprend le danois et ne parlera plus jamais sa langue maternelle. L'aumônier commence tout simplement par l'alphabet et l'écriture des lettres simples. Brique par brique, il bâtit une langue dans la tête du prisonnier et Gomez imite les sons avec un étonnement qui pourrait aussi bien être de la reconnaissance. La théorie de l'aumônier tient. Apparemment, Gomez ne réalise même pas qu'il a parlé une autre langue avant celle-ci. Et pour chaque nouveau mot qu'il maitrise, c'est un bout du monde qui renaitt (Le douzième cavalier, 42).

Gomez a oublié sa langue, mais il n'a pas oublié ses instincts, sa culture. Dans une description particulièrement crue, Ib Michael nous montre comment, en répétant les rites de ses aïeux indiens, il ôte la peau du gardien de prison et s'en sert littéralement comme d'un masque pour s'enfuir. Il passera le restant de ses jours, une vingtaine d'années, à errer au Danemark. De ville en ville, il triche, il se bat et vole pour gagner sa vie. Parfois, il se trouve en groupe mais dans le fond, il reste toujours seul, toujours hanté par le désir de bouger. Le monde de Gomez est un univers constamment en mouvement. Sa vie est un éternel voyage. Et ses enfants, surtout son fils issu du viol, renié par sa mère et adopté par une famille aisée de Copenhague, vont hériter de la nécessité constante de bouger, d'un perpétuel besoin de changement. Pour Gomez, ce besoin est inné, il s'est toujours déplacé, le voyage répond à un sentiment profondément ancré en lui. Vers la fin de sa vie, il rencontre la fille née de sa relation avec la servante, et c'est à sa fille qu'il laissera à sa mort la bible familiale avec un document en ancien maya qu'il ne sait pas lire. Ses dernières années, il les passe à 
chasser un trésor qui s'avère inexistant. Sa fille sera l'ancêtre de la famille danoise, l'aïeule du narrateur.

Quand nous quittons Gomez après les deux tiers du roman, c'est pour retrouver brièvement l'homme que nous connaissons déjà de La fille vanille. Il est toujours dans sa chambre d'hôtel à Roskilde. C'est lui qui à travers ses visions a reconstitué la vie de Gomez comme il fabriquait jadis les histoires grâce auxquelles il divertissait sa petite sœur. Il nous raconte qu'à sa mort sa grand-mère lui a laissé les lettres de son frère Viggo et le document maya de Gomez. La traduction de ce document, toujours inachevée, s'impose maintenant à lui, comme une priorité. Enfermé dans la chambre d'hôtel, il se sent pris au piège : J'ai retourné les lettres devant le miroir, j'ai encore le reste à traduire. J'ai le sentiment que je ne sortirai pas d'ici avant de l'avoir fait, (202-203). Et il poursuit : J'ai hérité d'un royaume de visions et d'objets trouvés. Quelque chose laisse croire que j'ai également hérité de la malédiction de la famille. Dans la première lettre de Viggo à grandmère [...], il écrit que "nos origines cachent des secrets qu'on ferait mieux de dissimuler à l'avenir ", (203). Ce qui se passe, ce qui s'est passé est donc le résultat du destin ; Gomez, l'oncle Viggo, le narrateur, sont menés par le destin.

Il en est de même pour le fils de Gomez, Edvard Emil Langberg. Elevé dans la haute bourgeoisie de Copenhague dans une famille adoptive, il apparait d'abord comme le fils exemplaire à l'avenir brillant, mais les brins de folie de son père se manifestent et se développent à tel point que son entourage en est heurté. Hanté par le gène voyageur de la famille et par l'amour impossible qu'il porte à la femme qu'il croit être sa sœur, il quitte le Danemark après la mort de celle-ci et part en Amérique. Ses finances épuisées, il vit de son violon et, à la recherche de son frère adoptif, il atterrit au Mexique. Le destin lui attribue vite le même sort qu'à son père : il devient mercenaire dans l'armée mexicaine. Dans ce passage, Ib Michael mêle encore une fois réalité et fiction en se servant du Danois Edvard Emil Langberg comme personnage romanesque. Non seulement il se sert de son nom mais également de l'histoire de sa vie. Car ce Langberg a vraiment existé, il a bien participé à la guerre d'Indépendance du Mexique dans les années 1860 et a failli devenir le président du pays (voir l'article Ib Michael enlève la peau, Berlingske Tidende, 28.3.1993). Il est cependant loin d'être le seul personnage historique à figurer dans ce roman. Ainsi l'aumônier de la prison ressemble fortement à l'écrivain danois Steen Steensen Blicher et dans le chapitre Bal masqué avec deux inconnus (161-169) figurent de nombreuses célébrités, tels les écrivains Hans Christian Andersen, Adam Oehlenschläger et Johan Ludvig Heiberg. Ib Michael obtient par ces détails non seulement une crédibilité mais aussi une certaine reconnaissance de la part de ses lecteurs car chacun reconnaît dans la description des personnages l'image qu'on s'est faite de ces écrivains qui font tous partie du «trésor national » danois. Dans Lettre à la lune, le même effet de reconnaissance est obtenu par des citations incorporées dans le texte, tels les mots d'une chanson connue par les Danois (Lettre à la lune, 182 par exemple) ou par d'autres allusions du même genre relevant d'un patrimoine commun à la plupart des lecteurs.

Pour Edvard Emil Langberg comme pour Gomez, le voyage apparaît comme l'expression de leur propre fébrilité. La vie de mercenaire leur sied parfaitement. Ce sont et le moment et les pulsions qui décident de l'orientation du voyage; le déplacement, le changement deviennent l'essentiel. Ainsi quand Edvard Emil Langberg rencontre la belle Séraphine et se marie avec elle, il ne cesse pas pour autant de bouger. Son destin est grandiose et tragique : il perd Rikke, sa sœur adoptive avant de savoir qu'elle n'est 
pas sa sœur véritable, il connaît dès ses années en collège sa vraie mère, mais quand elle choisit enfin de révéler sa propre identité dans une lettre que lui apporte son autre fils, il est trop tard. Langberg vient de mourir sur le champ de bataille. Son destin pourrait se résumer en une seule formule : malédiction familiale. Et c'est justement à cette malédiction que va revenir le narrateur, une fois les aventures de Langberg terminées. Je suis toujours là, client dans la chambre 17. C'est comme un film où personne - à aucun moment, n'arrive à quitter la chambre. À chaque fois que j'ai fait mes bagages quelque chose s'est passé. Le seuil vers le corridor est une frontière que je ne peux pas franchir (Le douzième cavalier, 266). Désormais, le voyage le plus difficile s'impose, l'affrontement absolu guette.

Le document hérité de Gomez date du $\mathrm{xV}^{\mathrm{e}}$ siècle et, malgré des études assidues, notre narrateur n'est pas encore parvenu à le traduire. Mais le moment est venu: Je veux sortir du cocon dans lequel ma famille m'a enlacé avec du fil de soie (270) déclare le narrateur.

Il est face à une grande tâche. La solution est le voyage dans les mots et les significations :

Puisque tous les mots-clés peuvent avoir jusqu'à douze significations différentes dans la traduction, je leur donne à chacun « un cavalier » que je peux faire partir dans mon texte. Ça s'appelle « rechercher-et-remplacer-par » dans mon traitement de texte. Quand je me sers de cette méthode et remplace pour la première fois un mot par un autre et que cela donne du sens dans plusieurs phrases de suite, je tremble comme un chien pisteur qui s'est arrêté devant sa proie. [...]. Je continue comme ça. [...]. L'un après l'autre, je fais partir mes cavaliers. Il neige sur l'écran en cristaux liquides, l'alphabet se dissout et se reconstruit. Au passage naissent de nouveaux signes où les éléments se mélangent furieusement; une écriture qui dure des secondes et qui ressemble à des gènes écroulés. Lentement, le document change de caractère. [...] À chaque minute, un nouveau morceau sort des membranes de la langue et devient chair sur le squelette, (270-271).

C'est dans ce passage que nous voyons clairement et plus que jamais la fonction et la raison d'être du voyage, du mouvement, dans l'œuvre d'Ib Michael. En premier lieu, il fait partie du destin inévitable, il est nécessaire au développement d'un homme, d'une famille, de l'Histoire. Il transforme le monde, il détruit et il construit. Il peut être à la fois une malédiction et une bénédiction comme nous le montre la suite de la trilogie: Lettre à la Lune. Le départ, l'absence, d'autres aspects du voyage, sont pour plusieurs des personnages de la trilogie des moyens de faire apprécier leur présence: ce n'est qu'après son départ en Amérique que la mère naturelle de Langberg se décide à lui dévoiler son identité, la mère du narrateur se fait beaucoup plus apprécier de son mari après son voyage, son échappée en Afrique, etc.. Le voyage possède de multiples facettes chez Ib Michael, il représente l'aventure, la découverte mais également la fuite. Il peut servir de délassement mais il peut également être une errance éternelle. Cette ambiguïté, nous en trouvons la source dans le document indien que le narrateur parvient enfin à traduire. Ici, Ib Michael donne libre cours au côté fantastique de son roman. Les destins se croisent et s'assemblent pour ne former qu'un seul fil. Le document a été écrit par Tecum, le premier ancêtre de la famille, l'homme qui est le fruit du viol d'un monde par un autre. Il est le fils de la reine des Indiens mayas et du chef des conquistadors espagnols. Tecum rédige ce document à l'article de la mort. Ses réflexions sur la vie, le destin, le malheur et le déchirement serviront de mots-clés pour nous expliquer et surtout pour expliquer au narrateur le sort de sa famille, le malheur et la malédiction qui pèsent sur sa lignée, les pulsions qui poussent les membres de la famille à agir, leurs envies, leur goût pour le déplacement, le changement et le voyage. 
L'auteur du document a vécu dans un monde profondément marqué par les ambiguïtés et les violences entre deux peuples très différents, celui de sa mère et celui de son père. Il fut lui-même le premier des enfants de la violence (277), il a passé son enfance en fuite avec sa mère mais s'est laissé séduire à l'adolescence par le monde de son père. À la mort de ce dernier, il a été rejeté de ce monde et des siens: Je devais pour toujours appartenir à la famille des traitres. Je cachai mon visage et devins voyageur [...]. Je n'avais plus de place sur terre et partout je voyais notre monde mourir, (280).

Dans ce document, nous rencontrons un thème central du roman : le jeu que mènent les dieux avec l'homme. Tecum le décrit dans les termes suivants: Aussi longtemps que je m'en souvienne nous avons eu des dieux. Nous leur avons fait des sacrifices. Mais si les dieux devaient se contenter des sacrifices des hommes, ils seraient devenus maigres. C'est pourquoi ils deviennent cruels au fil des ans et ne se soucient que d'eux-mêmes (272). Le narrateur fait référence à ce jeu à plusieurs reprises, par exemple quand il mentionne l'histoire d'amour impossible entre le demi-frère de Langberg et la veuve de Langberg : Ni l'un ni l'autre ne voulait la séparation; elle s'est perdue quelque part sur le chemin parce que les dieux se sont entre-temps désintéressés de leur propre jeu (265). C'est ce jeu qui explique les étranges tournures que prend parfois le destin d'une vie; le jeu des dieux sert d'image pour exprimer le fatalisme. L'autre élément important révélé par le document est le sort jeté par la mère de Tecum avant d'être violée par son futur père : Celui-ci fondera une famille où les pères mourront sans s'être réconciliés avec leurs fils et où les mères à chaque douzième lignée donneront naissance à un monstre (277). Le narrateur prend alors soudainement conscience du sort de Gomez: selon l'arbre généalogique de la famille (inscrit dans la Bible familiale), Gomez représente la douzième génération, il est le monstre. Le destin, la malédiction de la famille sont donc beaucoup plus prononcés chez Gomez, car il porte en lui le malheur et la malédiction : tout est plus violent, plus horrible, plus exorbitant chez Gomez. C'est cette découverte importante, la connaissance de la malédiction qui pèse sur sa famille qui amène le narrateur à la confrontation avec son propre malheur.

\section{Secrets, silences et absences}

Les thèmes importants de ces trois romans sont repris dans le dernier chapitre de Lettre à la lune et dans l'épilogue qui clôt la trilogie. Dans le dernier chapitre de Lettre à la lune, le narrateur révèle de quelle façon les documents, - le texte maya, les lettres de l'oncle Viggo et la Bible familiale - lui sont parvenus. Il nous narre son errance, le long voyage qui a précédé son retour à Roskilde. Il raconte comment il est parvenu à trouver l'île secrète de l'oncle Viggo abritant son navire, et il nous fait également part du temps qu'il a passé comme naufragé sur cette île, à découvrir, à dévoiler le secret familial. De façon symbolique, cette découverte est illustrée par la fragilité du navire. La découverte permet au narrateur de reconstruire l'histoire familiale et surtout de revoir son propre passé, son histoire et sa conception de soi. Il a besoin de détruire une partie du passé pour pouvoir en reconstruire un autre!

Jusqu'ici, le narrateur a lui-même contribué à maintenir le secret. Les histoires inventées dans son enfance contenaient toutes le silence, comme il le confirme luimême dans Le douzième cavalier: Je bâtis une tour d'histoires, puis - quand j'ai su écrire - je les notai. Chacune contenait le silence. Je suis le prisonnier d'un silence que ne sera pas rompu avant que la dernière porte du bâtiment ne claque (267). Ces dernières pages reviennent 
donc sur un élément clé du roman : le silence. Ce thème a beaucoup marqué la famille du narrateur mais surtout il a marqué son propre rapport avec le père. Avec ces trois romans qui révèlent progressivement le long récit de l'histoire familiale et la découverte de ses racines violentes, le narrateur parvient finalement à rompre la malédiction familiale, il arrive en fin de compte à faire la paix avec son père, une paix qu'il réussit à conclure justement grâce à son récit. Faire la paix avec son père, c'est aussi faire la paix avec lui-même. Par les liens du voyage avec la reconstitution du passé, l'affrontement avec tous les fantômes qui hantent le passé de la famille, le narrateur arrive à mieux se connaître, à mieux se comprendre et par la suite à mieux comprendre la relation difficile qu'il a eu avec son père. Il arrive même à avouer qu'il lui ressemble : Il courut - comme le soldat -, filait de son ombre avec la lune comme son unique guide. Jusqu'à ce que le dernier masque tombât et qu'il voie qu'il avait confondu son image avec celle de son père (Lettre à la lune, 379), écrit-il dans un texte, rédigé pour la lune vers la fin de Lettre à la lune. Une lettre qui souligne les thèmes principaux de la trilogie; le voyage, la narration et le silence. C'est un document sévère dans laquelle il se condamne pour le rôle qu'il a joué dans sa propre histoire, c'est une missive ironique qui laisse entendre que rien n'est vraiment important pour lui, qu'il a tout feint. La lettre dévoile toutes les craintes du narrateur, des craintes qu'il parvient seulement à supporter grâce à la distanciation, en usant de la troisième et non pas de la première personne. Mais les trois romans démontrent un bel équilibre entre le silence, l'absence, thèmes centraux dans la trilogie, et la forte présence de la narration. L'écriture transpire de cette présence, l'innovation dans l'écriture, les nombreux détails dans le récit, la langue vivante et multicolore, tout contribue à cette présence. Ainsi dans $L a$ fille vanille, nous découvrons le monde à travers les yeux de l'enfant. Effectivement, le voyage dans les souvenirs constitue le présent réel pour le narrateur. Ceci est fortement souligné par le fait que les souvenirs ont la force de se manifester physiquement dans la chambre d'hôtel. D'ailleurs, le récit se fait à la première personne du singulier tandis que les commentaires à propos du temps de la narration, par exemple dans le prologue et dans l'épilogue, se font à la troisième personne et donc avec une certaine distanciation.

L'épilogue souligne plus que tout qu'il n'existe pas une seule et unique réalité dans l'univers d'Ib Michael. Ayant terminé ce qu'il avait à faire, (1995, 381), l'homme sort de sa maison natale, ouvre le portail vers la rue et se retrouve sur l'île secrète de l'oncle Viggo! Plus que d'une réalité bien réelle, il s'agit donc plutôt d'une réalité mentale. Les dernières pages sur la vie dans l'île nous en apprennent beaucoup sur l'état mental du narrateur alors qu'il a enfin terminé ce qu'il avait à faire. La vie n'a pour un bref moment plus de sens pour lui, il préfère le grand vide, la décadence, il est saisi par l'envie de mourir, mais finalement, il reste là, sur l'île, à attendre un bateau, prêt pour un nouveau voyage! C'est dans cette idée, cette conception de son propre monde et de son avenir qu'il trouve enfin la paix. Le voyage est donc devenu une mode de vie pour lui. Il s'agit d'une conception souvent développée dans l'œuvre d'Ib Michael. Il en est de même par exemple pour les personnages de Kilroy, Kilroy et du Troubadour et la fille $d u$ vent. Le voyage y débute aussi comme une quête ou comme une fuite mais devient en fin de compte une condition de vie dont les personnages des romans ne voudront plus se passer, car Il est dur de se satisfaire de l'existant quand on a bu aux sources du changement (Le troubadour et la fille du vent, 228). 


\section{BIBLIOGRAPHIE}

\section{Euvres:}

Michael Ib, Brev til månen (Lettre à la lune), Copenhague, Gyldendal, 1995.

Michael Ib, Den tolvte rytter (Le douzième cavalier), Copenhague, Gyldendal, 1993.

Michael Ib, Vanillepigen (La fille vanille), Copenhague, Gyldendal, 1991 (traduction française paru en 1996 chez Christian Bourgois éditeur).

Michael Ib, Kilroy, Kilroy, Copenhague, Gyldendal, 1989.

Michael Ib, Troubadurens loerling (Le troubadour et la fille du vent), Copenhague, Gyldendal, 1984.

\section{Articles parus dans des journaux danois :}

Berlingske Tidende, AAGAARD, Lars Henrik, La Magie est partout, 8.12.1991.

Berlingske Tidende, AAGAARD, Lars Henrik, Ib Michael enlève la peau, 28.3.1993.

Information, Syberg, Karen, Ce n'est pas la vie qui est étrange, 2.4.1993.

Information, Skyum-Nielsen, Erik, Le Voyage jusqu'aux autres, 2.10.1995.

Jyllands-Posten, Grove, Gorm, La Vie est une aventure, 7.8.1993.

Jyllands-Posten, Juhl, Marianne, Un auteur devient adulte, 17.10.1995.

Politiken, Schierbeck, Ole, Insomnie heureuse, 28.3.1993.

Weekendavisen, Balsby, Egon, Une délivrance douloureuse, 6.10.1995.

\section{RÉSUMÉS}

Dans l'univers de la trilogie romanesque : La Fille vanille (1991), Le Douzième cavalier (1993) et Lettre à la lune (1995) de l'écrivain danois Ib Michael, le narrateur, de retour à Roskilde, sa ville natale, s'installe dans une chambre d'hôtel qui s'avère peuplée de fantômes, hantée par le passé et notamment par le passé de notre narrateur. Au centre de ce passé conflictuel se trouve le rapport du jeune homme avec son père, un rapport dont la source est la malédiction familiale. Ce n'est qu'après avoir traversé le labyrinthe que représente le passé mystérieux et exotique de sa famille, après avoir une fois pour toute affronté ses fantômes que le narrateur arrivera enfin à s'échapper, à faire la paix avec les fantômes, avec son père et avec soi-même. Le retour dans le passé est un long voyage spirituel qui va évoquer et faire vivre de nombreux autres voyages. Le narrateur revient en arrière dans l'histoire familiale et également dans l'Histoire avec un grand H. Le voyage débute comme une quête mais il devient en fin de compte une condition de vie dont le narrateur à la fin de son récit n'a plus envie de se passer.

Der dänische Verfasser Ib Michael schildert in seiner Trilogie «Das Vanillemädchen » (1991), «Der zwölfte Reiter» (1993) und «Brief an den Mond» (1995) in die Heimatstadt, Roskilde. Es stellt sich heraus, dass sein Hotelzimmer von Gespenstern der Vergangenheit bevölkert ist. Insbesondere sind es Gespenster aus der Vergangenheit des Verfassers, die ihn hier heimsuchen. 
Im Mittelpunkt dieser Vergangenheit steht das Verhältnis zwischen dem jungen Mann und seinem Vater, ein Verhältnis, dessen Probleme in einem alten Familienfluch tief vewurzelt sind. Erst als der Erzähler sich ein für allemal den Gespenstern der Vergangenheit seiner Familie gestellt hat, kann er seine Irrfahrten beenden. Dann kann er mit dem Vater, mit den Gespenstern, und mit sich selbst Frieden schliessen.

Seine Rückkehr in die Vergangenheit ist eine lange geistige Reise, die die Erinnerung an eine lange Reihe von anderen Reisen wachruft. Er reist weit umher, nicht nur in der Geschichte der Familie sondern auch in der Weltgeschichte.

Die Reise fängt als ein Suchen an, sie wird aber am Ende ein Bedürfnis, das der Verfasser nicht mehr entbehren möchte.

\section{AUTEUR}

\section{KIRSTEN-LAI JENSEN}

Université Charles-de-Gaulle - Lille 3 